\title{
Nursing-Sensitive Patient Care Outcomes in Taiwanese Nursing Homes
}

\author{
Ke-Ping A. Yang, Lillian M. Simms, Jeo-Chen T. Yin
}

Rapid social and economic development, changes in life style, aging of the population, changes in disease patterns, and the increasing threat of imported infectious diseases in Taiwan, the Republic of China have contributed to recent changes in the focus of health care. To meet the needs of this changing society, the National Assembly in 1992, amended the constitution stipulating that "The province shall implement a national health insurance program and promote research and development of modern and traditional medicines" (DOH, 1993). A philosophic shift from disease prevention to health promotion has created an opportunity for professional nursing to examine and evaluate its practice. Furthermore, the increase in number of nurse managed nursing homes has afforded a health care setting where outcomes of patient care are more likely to be sensitive to the effects of nursing care. Our recently completed research was designed to explore factors contributing to nursing-sensitive patient care outcomes in eight selected nursing homes in Taiwan.

The purpose of the study was to measure the effect of nursing care on biopsychosocial functioning of nursing home patients using instruments designed to: determine predictors of patients' biopsychosocial functioning; identify nursing practice patterns of care providers; assess level of patient satisfaction with care; and to identify the degree to which individual and organizational factors have an effect on biopsychosocial functioning. Related research questions were addressed with descriptive statistics and key hypotheses were generated and tested using inferential statistics. The researchers hypothesized that individual demographic factors, patient satisfaction, and organizational factors would significantly affect nursing care outcomes including improving the level of biopsychosocial functioning.

The study was guided by a framework for patient-care outcomes that assumes that patient biopsychosocial functioning is affected by individual and organizational factors. The framework was adapted from Beckman and Simms (1992) in their work on redesigning nursing practice patterns. Data for the study were collected from 224 nursing home patients and 8 nursing home executives. Patients' information was collected through a three-part questionnaire. Participating nurse executives completed a data form about the home's nursing staff and organizational characteristics. Pilot testing of a Chinese version of the instruments was performed not only to check the quality of translation, but also for the practical aspects of test administration.

Biopsychosocial functioning was measured by the Biopsychosocial Functioning Scale (BFS) which was adapted from the Sickness Impact Profile (SIP) and Health Status Questionnaire (HSQ) (Yang, 1995). Perceived satisfaction with care was measured by a questionnaire exploring eight dimensions of satisfaction including amount of care received, support and understanding from caregiver, trust relationship with caregivers, staff attitude and manner, confidence in professional ability of staff, freedom from tension, and overall life satisfaction in the nursing home. A single-item question was used to score subjective states of patients' perceived quality of care. The Organizational Characteristics Questionnaire consisted of 11 items pertaining to qualifications of nurses, practice patterns, and admission criteria.

Patients in the study were predominately mainlanders, the majority were male, illiterate widowers, Buddhist, and retired military personnel. The findings' were as follows: (a) Individual factors significantly predicted patients' biopsychosocial functioning, whereas organizational factors were generally not significant; (b) Both the single-item quality of care and patient satisfaction significantly affected functioning, but the two were not related; (c) Nursing practice patterns alone did not directly affect biopsychosocial functioning, but did so indirectly through satisfaction with care; (d) Subjects' social and psychological functioning, and their social and physical functioning were positively and significantly related, yet there was no significant relationship between physical and psychological functioning; (e) Positive changes occurred in patients' health conditions during their nursing home stay regardless of the nursing practice pattern.

Our research raised many questions; two interesting factors stand out. Rarely are physicians or other professionals involved in direct patient care in the nursing homes. Secondly there was no clear single nursing practice pattern. However, similar nursing practices were observed in all settings: nurses provided skilled nursing, rehabilitation, and health education, and were in charge of the units; nurse-aides provided most of the basic nursing care. All RNs were women, with an educational level of junior college or vocational nursing school and all had more than 5 years of nursing experience. Positive changes occurred in participants' health conditions during their stay in these settings leading the researchers to assume that additional environmental factors should be assessed and measured.

\section{References}

Beckman, J.S., \& Simms, L.M. (1992) . A guide to redesigning nursing practice patterns. Ann Arbor, MI: Health Administration Press.

DOH (Department of Health). (1993). Public health in Taiwan area, Republic of China. Republic of China: The Executive Yuan.

Yang, K.P. (1995). Predictors of nursing-sensitive patient outcomes in longterm care facilities in Taiwan, ROC. Doctoral Dissertation, the University of Michigan, Ann Arbor, MI.

Image: Journal of Nursing Scholarship, 1998; 30:3, 290. (C)1998 Sigma Theta Tau International.

[Key words: outcome evaluation; nursing-sensitive patient outcomes; biopsychosocial functioning] Ke-Ping A. Yang, RN, PhD, Lambda Beta-At-Large, Nursing Supervisor and
Associate Professor, Taichung Veterans General Hospital and Chung-Shan
Medical College, Taichung, Taiwan. Lillian M. Simms, PhD, FAAN, Rho,
Associate Professor of Nursing Emeritus, Nursing Administration and Health
Gerontology, University of Michigan, Ann Arbor, MI. Jeo-Chen T. Yin, RN,
PhD, Lambda Beta-At-Large, Provincial Commissioner of Taiwan and Asso-
ciate Professor, National Yan-Ming University, Taiwan. The authors acknowl-
edge this study was supported by the National Science Council, Republic
of China. Special recognition goes to Deborah Oakley, Professor, Center
for Nursing Research; Irene Butter, Professor of Public Health Policy; and
Richard Redman, School of Nursing; all at the School of Nursing, Univer-
sity of Michigan for their contribution to the development of this study.
Correspondence to Dr. Yang, Department of Nursing, Taichung Veterans
General Hospital, 160, Chung-Kung Rd., Sec. 3, Taichung, Taiwan 40705.
Accepted for publication December 3, 1997. 